\title{
A Study on Nonlinear Equivalent Circuit for the Electro-impulse De-icing System
}

\author{
Yuanyuan $\mathrm{Xu}^{1}$, Hongjuan $\mathrm{Ge}^{2}$, Peng $\mathrm{Chen}^{3}$ \\ ${ }^{1,2,3}$ College of Automation, Nanjing University of Aeronautics and Astronautics

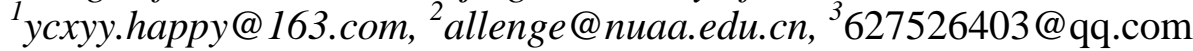

\begin{abstract}
A nonlinear equivalent circuit method was proposed for studying and designing the EIDI (electro-impulse de-icing) system more effectively. After analyzing the impulsive force of skin, nonlinear equivalent load of the EIDI system which on behalf of the vibrational energy was analyzed by using the law of conservation of energy, and nonlinear equivalent circuit model related to the system parameter was established. Firstly, a three-dimensional electromagnetic field model for modeling the axisymmetric ring-type pulsed coil was presented, which was based on finite element simulation software Ansoft Maxwell , and the expression of the normal electromagnetic force during the complete deicing cycle was obtained by numerical fitting method. Secondly, the deformation expression of the aluminum skin induced by the normal electromagnetic force was computed, then the nonlinear equivalent circuit model was researched based on the work done by the electromagnetic force. Finally, the circuit parameter in the different coil-aluminum-plate gap was designed by using nonlinear equivalent circuit model, this parameter was consistent with experiment parameters, which verifies the correctness of the circuit parameters and provides the theoretical basis for the EIDI system.
\end{abstract}

Keywords: Nonlinear Equivalent Circuit, Electro-impulse De-icing System

\section{Introduction}

Aircraft icing was a nature phenomenon that the water vapour on the aircraft surface condenses into ice in the specific meteorological conditions. Time and time again in the history of aviation, there are so many accidents caused by aircraft icing, therefore, the aircraft icing phenomenon and the studying of anti-icing and de-icing which should be seriously considered was the important issue of safe flight.

At present, there are mainly three kinds of de-icing method, cryoscopy, hot melt and surface deformation[1], in the aircraft de-icing system. In the cryoscopy method, after spraying anti-icing fluid, the freezing point of mixed liquid will be lower than the water so as to prevent the formation of ice. However, this method was not applicable to the ice with strong adhesion strength. Hot melt method by which heat could be generated to make the ice melt, evaporate or sublimate directly, was used in the ARJ21-700 regional aircraft to deice the wings and engine inlet[2], nevertheless, deicing with the engine bleed air will reduce the efficiency of the engine, increase fuel consumption and reduce the overall rate of heat energy utilization. Surface deformation method which belongs to the mechanical deicing can be used to make the skin surface deformation and the ice on the surface will disrupt and fall. Electro-impulse de-icing one of the surface deformation method was more mature in Russia[3], which has the characteristics of light weight, small volume, low power consumption and easy to repair. So this method will have a good application prospect in all electric aircraft in the future.

EIDI system of three generations have been developed since the early 1920s in Russia, which were Installed in the Ilyshin series aircraft and well run. Then, American FAA established a project team based on Wichita State University, which has developed source 
code taking the capacitor voltage as the input to study the de-icing incentive[4-7]. The EIDI technology was developed late in our country, the electrodynamics model was built by Beijing University of Aeronautics and Astronautics to predict the de-icing area[8-11]. Nanjing University of Aeronautics and Astronautics has established a FE model of system eddy current, at the same time ANSYS software was used to analyze the failure element of skin surface[12-14]. Also, Northwestern Polytechnical University has researched the EIDI system, and attempted to study the factors of de-icing system with the solutions of electromagnetic eddy current field[15].

Based on the three-dimensional transient magnetic field simulation model of EIDI system, the work of normal electromagnetic force was studied in the deicing incentive cycle, meanwhile, a nonlinear equivalent circuit related to the parameters of EIDI system was established. At last, the circuit parameters of energy storage elements in different air gap were designed under the equivalent circuit model.

\section{EIDI System}

The principle circuit of EIDI system was shown in Figure 1. When the thyristor was triggered to turn-on, the capacitor will recharge the pulsed coil. Because the resister and inductance of this pulsed coil was very small, an excessive current only for short periods will be sent through the coil, which lead to the transient magnetic field around the coil. Due to the gap between the coil and the aluminum skin was very small, A large eddy current will be induced by the transient magnetic field in aluminum skin. Eddy current and transient magnetic field, these two interact with each other to produce short electromagnetic force, the peak of which can reach hundreds or even thousands Newton. Strong acceleration generated from the electromagnetic force can make the ice on the wing surface peeling off, and the effect of deicing can be ultimately achieved. The diode connected in anti-parallel with the capacitor was used to prevent the capacitor reverse charging, and prolong the capacitor 's life.

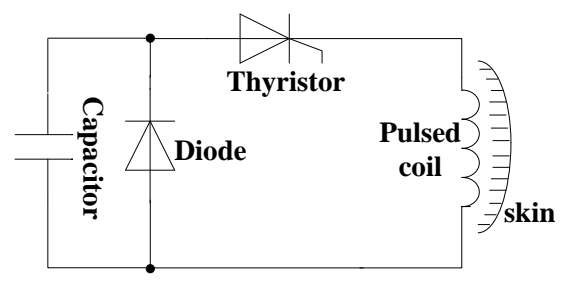

\section{Figure 1. Diagram of Electro-impulse De-icing (EIDI) System}

\section{Analysis and Calculation of the Skin Pulse Force}

\subsection{Analysis of Transient Magnetic Field for Pulsed Coil}

Figure 2 was the simplified model of electromagnetic field in the EIDI system, pulsed coil was the axisymmetric cylindrical hollow coil, which was wound by copper belt.

In cylindrical coordinates $(\rho, \varphi, \mathrm{z})$, the monolayer copper tape is wound by the copper coils of 1,000 turns, the plane of each coil parallel with the xoy plane, the radius of the coil is a, the current pass through the coil is $I_{0} / T$, the width of copper tape is $b_{\text {width }}, \mathrm{N}$ shows how many coil turns in a unit width $\left(N=T / b_{\text {width }}\right)$, the center coordinates of the coil is $(0,0, h)$, the coordinates of each field points in $Z_{0}$ plane is $\left(\rho_{o}, \varphi_{o}, Z_{o}\right)$.

The magnetic vector potential produced by the field source with uniform current distribution is: 


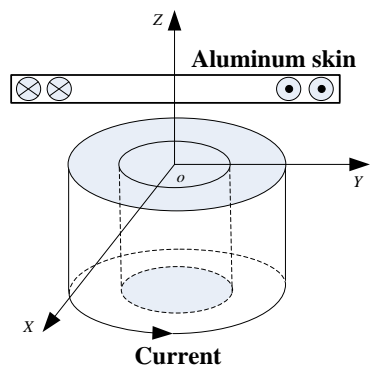

Figure 2. The Electromagnetic Field Simplified Model of the EIDI System

$$
A=\frac{\mu_{0} I_{0}}{4 \pi} f_{l} \frac{d l}{R}
$$

$l$ is the ring loop, $d l$ is the tangent vector of every point in the ring wire, $\mathrm{R}$ is the vector from point $\mathrm{Q}$ in ring to each point $\mathrm{P}$. With the cylindrical coordinate system transformation, the magnetic vector potential of single turn coil in each $\mathrm{Z}_{0}$ plane is:

$$
A=\left\{\begin{array}{l}
0, \rho_{0}=0 \\
A_{\varphi} e_{\varphi}=\frac{\mu_{0} I_{0}}{2 \pi T} \sqrt{\frac{a}{\rho_{0}}} f(k) \mathrm{e}_{\varphi}, \rho_{0}>0
\end{array}\right.
$$

In which,

$$
\begin{gathered}
f(k)=\left(\frac{2}{k}-k\right) K(k)-\frac{2}{k} E(k) \\
k=\sqrt{\frac{4 \rho a}{\left(\rho_{0}+a\right)^{2}+\left(Z_{0}-h\right)^{2}}} \\
K(k)=\int_{0}^{\frac{\pi}{2}} \frac{d \alpha}{\sqrt{1-k^{2} \sin ^{2} \alpha}} \\
E(k)=\int_{0}^{\frac{\pi}{2}} \sqrt{1-k^{2} \sin ^{2} \alpha} d \alpha
\end{gathered}
$$

$K(\mathrm{k})$ and $E(\mathrm{k})$ represent the elliptic integral of the first and second kind with modulus $\mathrm{k}$ and modular angle,$e_{\phi}$ is the circumferential tangential unit vector. Based on the rotation formula of cylindrical coordinates:

$$
B=\nabla \times A=B_{\rho} e_{\rho}+B_{z} e_{z}
$$

In this function, $e_{\rho}$ and $e_{Z}$ represent the unit vector in the direction of $\rho$ and $Z$ in cylindrical coordinates, so radial magnetic induction of single copper tape is:

$$
B_{\rho}=\frac{\mu_{0} I_{0}}{\pi b_{\text {width }}} \sqrt{\frac{a}{\rho_{0}}}\left[\left(1-\frac{1}{2} k^{2}\right) K(k)-E(k)\right]
$$

Axial magnetic induction is:

$$
B_{Z}=\frac{\mu_{0} I_{0}}{2 \pi b_{\text {width }}}\left[g_{z}\left(\rho_{0}, \mathrm{z}-\mathrm{z}_{2}\right)-g_{z}\left(\rho_{0}, \mathrm{z}-\mathrm{z}_{1}\right)\right]
$$

$Z_{1}$ and $Z_{2}$ are the height of two end faces at the top and bottom of the pulse coil, namely the axial magnetic induction of pulsed coil is the sum of each axial magnetic induction of single copper tape with different radii.

As shown in Figure 3, three-dimensional simulation model of the EIDI system was established by the Ansoft finite element analysis software, the model parameters are shown in Table 1. When studying the electromagnetic force acting on the aluminum skin, because of the complexity of EIDI system, the other parameters and installation conditions remain unchanged, only a single parameter changed. The trends of radial 
electromagnetic peak force and normal electromagnetic peak force are studied when the gap between the coil and the skin changed.

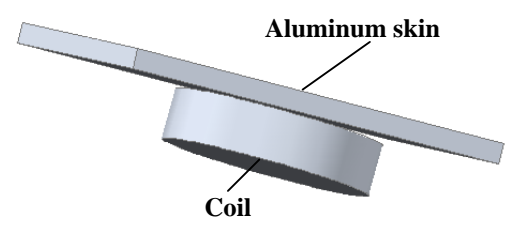

Figure 3. Three-dimensional Simulation Model of the EIDI System

Table 1. Parameters of the EIDI System Simulation Model

\begin{tabular}{cccccc}
\hline $\begin{array}{c}\text { The circuit parameters } \\
\text { of pulsed coil }\end{array}$ & $\begin{array}{c}\text { The structure parameters } \\
\text { of pulsed coil }\end{array}$ & \multicolumn{2}{c}{$\begin{array}{c}\text { The structure parameters } \\
\text { of aluminum skin }\end{array}$} \\
\hline Inductance $/ \mathrm{uH}$ & 132 & Outside diameter $/ \mathrm{mm}$ & 30 & Length*Width/mm & $100^{*} 100$ \\
Resistance $/ \Omega$ & 0.2 & Inside diameter $/ \mathrm{mm}$ & 5 & Air gap $/ \mathrm{mm}$ & 1 \\
- & - & Height $/ \mathrm{mm}$ & 10 & Thickness $/ \mathrm{mm}$ & 1 \\
\hline
\end{tabular}

\subsection{The Simulation and Research of Impulsive Force for Aluminum Skin}

3.2.1. The influence of coil-skin gap on the peak of pulse-electromagnetic force: The change of the gap between aluminum skin and pulsed coil will cause the variation of pulse-electromagnetic force. So with the excitation current and the other parameters unchanged, only adjust the gap between the pulsed coil and the skin. When the gap is $0.5 \mathrm{~mm}, 1.0 \mathrm{~mm}, 1.5 \mathrm{~mm}, 2.0 \mathrm{~mm}, 2.5 \mathrm{~mm}, 3.0 \mathrm{~mm}$, the force effect on the aluminum skin was analyzed. The relationship between radial/normal electromagnetic peak force and the skin-coil gap was shown in Figure 4(a)

With reference to Fig .4(a), the normal electromagnetic peak force will decrease with the increase of gap, and the radial electromagnetic peak force will go up in proportion to the gap.

3.2.2. The influence of skin thickness on the peak of pulse-electromagnetic force: Because of the skin effect, skin thickness will affect the pulse force of aluminum skin to a certain extent. According to the formula for calculating the skin depth, $3.5 \mathrm{~mm}$ is the best value for the aluminum skin depth in the working conditions. Under these conditions that the excitation current and other conditions of de-icing system are not changed, the force effect on the aluminum skin was analyzed only when the skin thickness was changed. As shown in Figure 4(b), the radial/normal electromagnetic peak force will vary with the skin thickness, which was $1 \mathrm{~mm}, 1.5 \mathrm{~mm}, 2.0 \mathrm{~mm}, 2.5 \mathrm{~mm}, 3.0 \mathrm{~mm}, 3.5 \mathrm{~mm}, 4.0 \mathrm{~mm}$ or $5.0 \mathrm{~mm}$.

Figure 4(b) represents that the normal electromagnetic peak force will go up in proportion to the skin thickness when the skin thickness was less than the skin depth, however in the condition the skin thickness was greater than the skin depth, the change of the normal electromagnetic peak force was obscure. While the peak of radial electromagnetic force was increase with the increase of the skin thickness. 


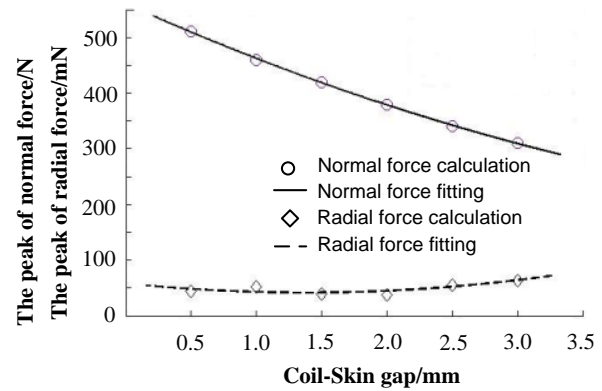

(a) coil-aluminum skin gap changed

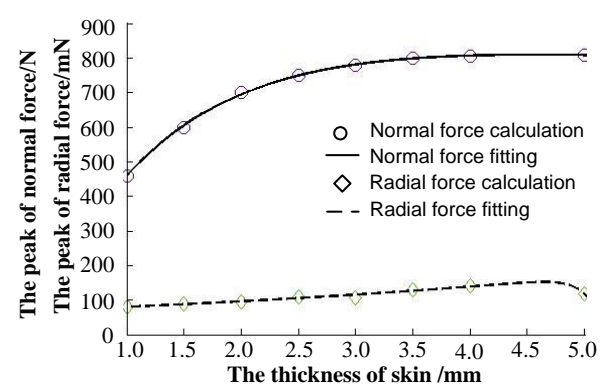

(b) the skin thickness changed

\section{Figure 4. The Electromagnetic Force Peak versus Coil-aluminum-plate Gap and Thickness of the Aluminum Plate}

Figure 4 (a) and Figure 4 (b) shows that no matter the skin thickness or the coil-skin gap changes, the peak of radial electromagnetic force compared to normal electromagnetic peak force changed little which was less than 0.5 percent, so the pinch effect of pulsed coil in the horizontal direction caused by the radial electromagnetic force can be ignored, namely the electromagnetic force all acted on the aluminum skin will produce vibratory motion with a small amplitude and strong acceleration to achieve the effect of deicing.

\section{Analysis of Nonlinear Equivalent Circuit}

\subsection{The Calculation of Work of Electromagnetic Force}

From the analysis in the 2.2 section, when analyzing the impulsive force generated by the pulsed magnetic field, the normal electromagnetic force was the only force which should be considered. When the excitation current shown in Figure 5 flows through the pulse coil, the electromagnetic force effect on the aluminum skin are shown as follows.

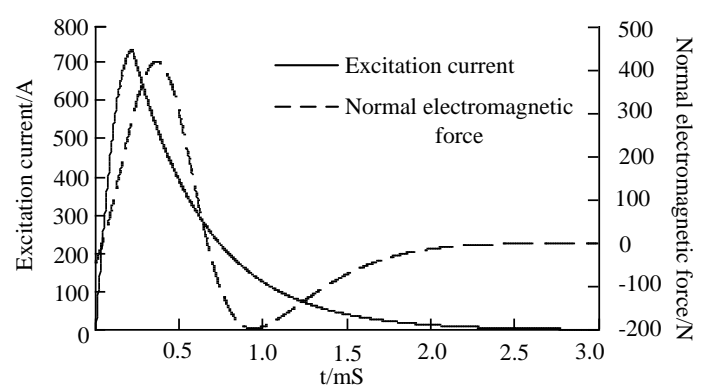

\section{Figure 5. Curves of Excitation Current and the Electromagnetic Force}

According to the curve fitting method, the expression of the relation between electromagnetic force and time is as follows.

$$
\left.F_{N}(\mathrm{t})\right|_{d=1}=-249.4 e^{-\left(\frac{t-0.4785}{0.8535}\right)^{2}}+719.2 e^{-\left(\frac{t-0.3675}{0.3348}\right)^{2}}
$$

In this expression, $d$ is the gap between the aluminum skin and pulsed coil.

After the electromagnetic force was applied at the aluminum skin, the shape of skin will change. According to the related knowledge of material mechanics, the deformation of metal skin caused by the electromagnetic force was equivalent to the deformation in the simply supported beam model shown in Figure 6. 


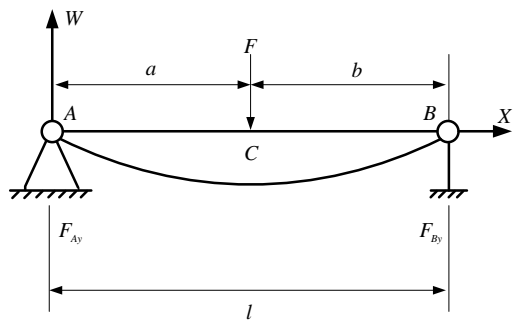

\section{Figure 6. Deformation Calculation Model of the Aluminum Skin}

Assuming the force acting on the skin focuses on the geometric centre point, when $a=b=l / 2, l$ is the length of aluminum skin, the maximum deformation of the skin is as follows.

$$
D=-\frac{F_{N}(\mathrm{t}) l^{3}}{48 E I}
$$

Where EI is the flexural rigidity of aluminum plate(a constant), which can be found in the related materials handbook.

Then the work of electromagnetic force in the deicing incentive process is:

$$
W_{F_{N}}=\int\left|F_{N}(\mathrm{t})\right| \cdot d D=\frac{l^{3}}{48 E I} \int\left|F_{N}(\mathrm{t})\right| \cdot \frac{d F_{N}(\mathrm{t})}{d t}
$$

\subsection{The Study on Nonlinear Equivalent Circuit}

Based on the principle of energy conservation, the formula of calculating electrical work of the exciting current in a complete period, which is equal to the energy consumption process of the nonlinear resistive load, is derived as follows:

$$
W_{F_{N}}=\int i^{2} R_{\text {equ }} \cdot d t
$$

Figure 7 shows the equivalent operation modal of pulsed discharge circuit, including discharging and freewheeling two stages. The freewheeling was to prevent reverse charging capacitor group and extend the service life of the capacitor. In this figure, $C$ is the capacitor group for storing energy, $L$ is the inductance of $\operatorname{pul}_{R} \operatorname{sed}_{R}$ coil, $R$ is the sum of the resistance of nonlinear resistive load and pulsed coil $\left(R=R_{\text {equ }}+R_{\text {coil }}\right), D$ is the freewheeling diode. Because the magnetic field generated by the eddy current of skin has low impact on the exciting current, the influence of magnetic field on the circuit and the eddy current loss of the pulsed coil can be ignored.

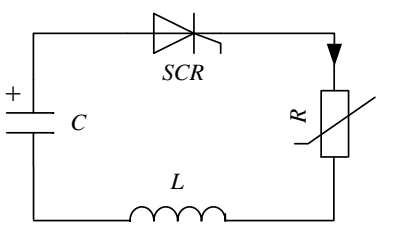

(a) discharging

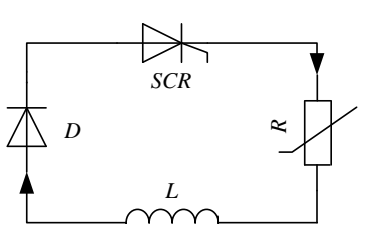

(b) freewheeling

Figure 7. Operational Model of the EIDI System

In Figure 7 (a), according to Kirchhoff's voltage law and the solving method of nonlinear circuit, the expression can be got as follows: 


$$
\left\{\begin{array}{l}
L C \frac{d^{2} U}{d t^{2}}+R C \frac{d U}{d t}+U=0 \\
\left.U\right|_{t=0}=U_{0} \\
\left.\frac{d U}{d t}\right|_{t=0}=0
\end{array}\right.
$$

Because the resistance of pulsed coil is very small, $R<2 \sqrt{L / C}$, the circuit operates in the underdamping state, the voltage across the capacitor in the discharging process is:

$$
U=\frac{U_{0}}{\omega \sqrt{L C}} e^{-\delta \cdot t} \sin (\omega t+\varphi)
$$

In this expression,

$$
\delta=\frac{R}{2 L}, \omega_{0}=\frac{1}{\sqrt{L C}}, \omega=\sqrt{\omega_{0}^{2}-\delta^{2}}, \varphi=\arcsin \left(\frac{\omega}{\omega_{0}}\right)
$$

Also , the current flowed through the pulsed coil without regard to the freewheeling diode is:

$$
i=-\frac{U_{0}}{\omega L} e^{-\delta \cdot t} \sin \omega t
$$

So the peak current flowed through the pulsed coil is:

$$
i_{\text {max }}=\frac{U_{0}}{\omega L} e^{-\delta \cdot t_{i, \max }} \sin \theta
$$

And the corresponding time to peak current:

$$
t_{i, \max }=\frac{\theta}{\omega}, \quad \theta=\arctan \left(\frac{\omega}{\delta}\right)
$$

Therefore, considering the existence of anti-parallel freewheeling diode, the discharging current is:

$$
i=\left\{\begin{array}{l}
\frac{U_{0}}{\omega L} e^{-\delta \cdot t} \sin \omega t, 0 \leq t \leq t_{i, \text { max }} \\
i_{\max } e^{-\frac{R}{L}\left(t-t_{i, \max }\right)}, t>t_{i, \max }
\end{array}\right.
$$

Based on the expression (9), (11), (12), (13) and (14), the nonlinear equivalent resistance is:

$$
R_{e q u}=\left\{\begin{array}{l}
\frac{\left|\mathrm{F}_{N}(\mathrm{t}) \cdot \mathrm{F}_{N}^{\prime}(\mathrm{t})\right| l^{3}}{48 E I \cdot U_{0}^{2}} \cdot \frac{\left(\frac{4 L}{C}-R^{2}\right) \cdot e^{\frac{R}{L} \cdot t}}{\sin ^{2} \omega t}-R_{c o i l}, 0<t \leq t_{i, \max } \\
\frac{\left|\mathrm{F}_{N}(\mathrm{t}) \cdot \mathrm{F}_{N}^{\prime}(\mathrm{t})\right| l^{3}}{48 E I \cdot U_{0}^{2}} \cdot e^{\frac{R}{L}\left(t-\frac{\theta}{\omega}\right)}-R_{c o i l}, t>t_{i, \max }
\end{array}\right.
$$
8.

In the above case, the nonlinear equivalent circuit of EIDI system is shown in Figure

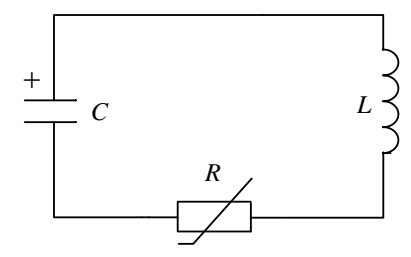

\section{Figure 8. Nonlinear Equivalent Circuit of the EIDI System}

Figure 9(a) is the nonlinear equivalent resistance-air gap between aluminum skin and pulse coil curve, and Figure 9(b) is the air gap-electrical work of electromagnetic force in 
a period curve. In these two figures, the curve 1-5 identify the air gap is 1.0, 1.5, 2.0, 2.5 and $3.0 \mathrm{~mm}$.

It can be seen from the Figure 9, the nonlinear equivalent resistance and the electrical work of electromagnetic force in a period will decrease with the increase of the air gap.

Because the maximal capacity usage ratio $\eta$ of EIDI system is less than $30 \%, \eta_{\text {is }}$ the ratio of electrical work of electromagnetic force and stored energy of the capacitor, the following expression can be derived.

$$
\frac{1}{2} C U_{0}^{2} \cdot \eta=\int i^{2} R_{e q u} \cdot d t
$$

Which, $C=\frac{100 \int i^{2} R_{e q u} \cdot d t}{32 \eta} u F$.

For the expression (16), $U_{0}$ is set as $800 \mathrm{~V}$ by empirical value. At the same time, according to the electrical work of nonlinear equivalent resistance, the capacitor value takes its maximum. The suggested values of stored energy element $\mathrm{C}$ under the different air gap are shown in Table 2.
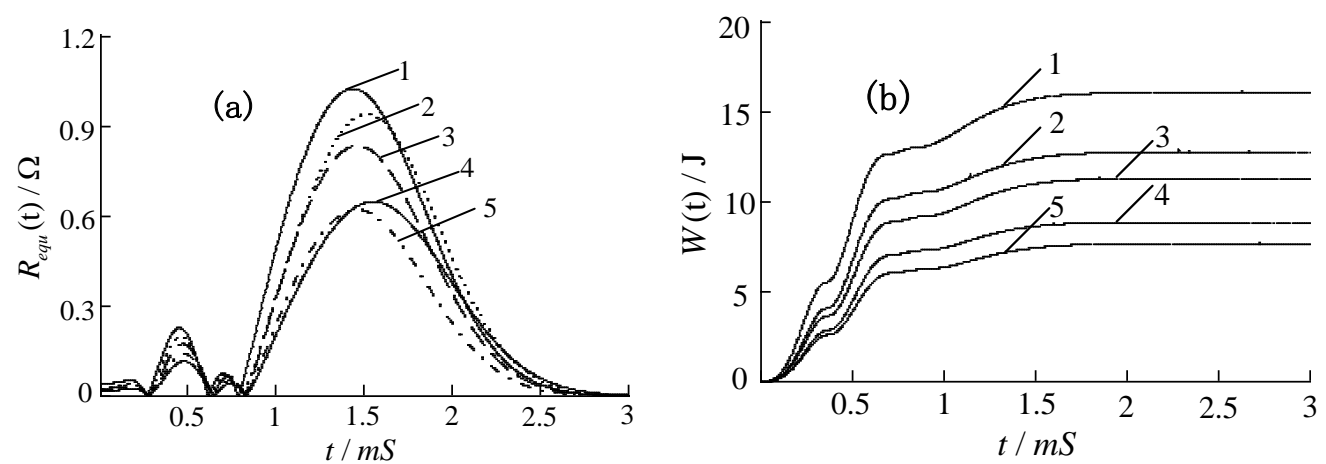

Figure 9. Curves under Different Gap

Table 2. Energy Storage Element Selected under Different Gap

\begin{tabular}{ccccc}
\hline $\begin{array}{c}\text { Air } \\
\text { gap/mm }\end{array}$ & $\begin{array}{c}\text { nonlinear equivalent } \\
\text { resistance } / \Omega\end{array}$ & $\begin{array}{c}\text { Curves of electrical } \\
\text { work in a cycle /J }\end{array}$ & $\begin{array}{c}\text { suggested } \\
\text { capacitor values } \\
\text { /uF }\end{array}$ & $\begin{array}{c}\text { energy } \\
\text { utilization } \\
\text { ration/\% }\end{array}$ \\
\hline $\mathrm{d}=1.0$ & Figure 9(a) Curve 1 & Figure 9(b) Curve 1 & 180 & 27.8 \\
d=1.5 & Figure 9(a) Curve 2 & Figure 9(b) Curve 2 & 212 & 22.1 \\
d=2.0 & Figure 9(a) Curve 3 & Figure 9(b) Curve 3 & 240 & 19.5 \\
d=2.5 & Figure 9(a) Curve 4 & Figure 9(b) Curve 4 & 306 & 15.3 \\
d=3.0 & Figure 9(a) Curve5 & Figure 9(b) Curve 5 & 352 & 13.3 \\
\hline
\end{tabular}




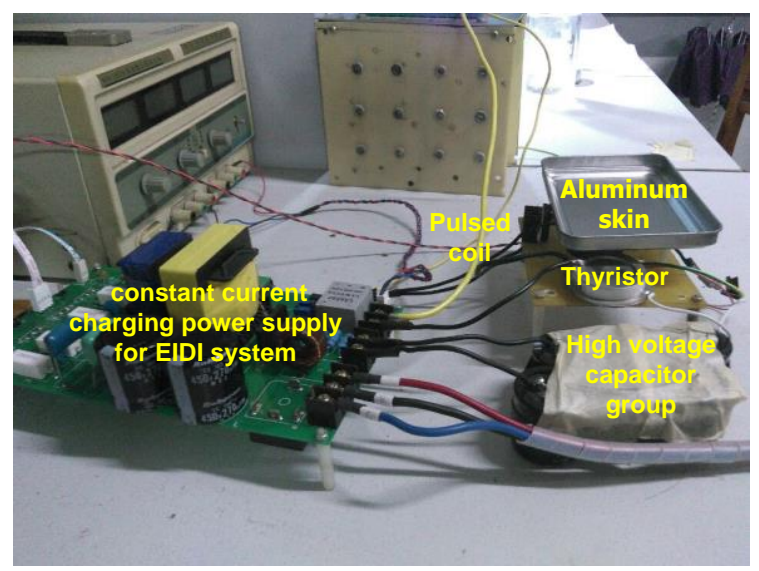

Figure 10. Test Platform of the EIDI System

Figure 10 shows the structures of the EIDI experiment platform, the thickness of the pulse coil is $0.2 \mathrm{~mm}$, which is wound by $10 \mathrm{~mm}$ copper belt and installed under the aluminum skin. The air gap between the aluminum skin and pulsed coil is adjusted flexibly with the experimental demand. This experiment platform is used to validate the consistency between the stored energy capacitor values in different air gap and the values suggested in Table 1. If the results are same, the accuracy of the method can be validated.

\section{Conclusion}

From the above analysis, simulation and experiment, the nonlinear equivalent circuit method could effectively reduce the difficulty of design the EIDI system. Based on the three-dimensional electromagnetic field simulation model of EIDI system, the electrical work of electromagnetic force in a whole excitation process was researched. The nonlinear equivalent circuit of EIDI system was analyzed with the principle of energy conservation, and the theoretical expression of nonlinear equivalent resistance was derived. Also, the values of capacitor of the EIDI system with different air gap was given by using the circuit model, which was consistent with the experimental data, the correctness of this method was verified.

\section{References}

[1] Li Qingying. Research on the experiments, theories, and design of the electro-impulse de-icing system.Nanjing:Nanjing University of Aeronautics and Astronautics,(2012):1-157.

[2] Du Qian.Design and research on electro-impulse de-icing system.Nanjing: Nanjing University of Aeronautics and Astronautics, (2009):1-71.

[3] Levin I.A.A device for removing ice from surfaces of thin-walled structures U.S:3,809,341, May, (1974).

[4] Zumwalt G W, Scharg R L,Benhart W D, et al. Analysis and tests for design of an electro-impulse deicing system. NASA Contractor Report 174919, (1985).

[5] Zumwalt G W,Scharg R L,Benhart W D,et-al.Electro-impulse de-icing testing analysis and design .NASA CR-4175,(1998).

[6] Schrag R L,Benhart W D,Electro-impulse de-icing electrodynamics solution by discrete elements. AIAA 26th Aerospace Sciences Meeting,Reno,Nevada:AIAA,(1988).

[7] Benhart W D, Gien P E.A structural dynamics investigation related to EIDI applications.AIAA Paper 860550,(1986).

[8] Li Guangchao,He Jiang,Lin Guiping,Electro-impulse de-icing (EIDI) technology study. Journal of Aerospace Power, vol. 26, no. 8, (2011), pp.1728-1736.

[9] Zhong Guo,Cao Yihua.Numerical simulation of ice accretion on multiple element airfoil and prediction on antiice situation. Journal of Beijing University of Aeronautics and Astrnautics, vol. 37, no. 1,(2011),pp. 36-40.

[10] Wang Chao, Chang Shinan, Yang Bo, Li Mingzhong.Investigation of runback ice during aircraft antiicing process.Journal of Beijing University of Aeronautics and Astrnautics, no. 6, (2013),pp. 776-781. 
[11] Mei Zhongyi,Gao Hong,Wang Yunqiao.Analyzing and controlling distortion of aircraft aluminium alloy structural part in NC machining. Journal of Beijing University of Aeronautics and Astrnautics, vol. 35, no. 2,(2009),pp. 146-150.

[12] Li Qingying,Zhu Chunling,Bai Tian,De-icing experiment and numerical simulation of the electroimpulse de-icing system. Journal of Aerospace Power, vol. 27, no.2,(2012),pp.350-357.

[13] Li Qingying,Bai Tian,ZHU Chuling.Electromagnetic field analysis for electro-impulse de-icing system[J],Journal of Nanjing University of Aeronautics \& Astronautics, vol. 43, no. 1,(2011),pp.95-100.

[14] Liang Qingsen, Chen Weijian, Ma Hui, Zhang Dalin, Injection Performance of Hot-Air De-icer with Micro-Injector[J]. Journal of Nanjing University of Aeronautics and Astrnautics. vol. 45, no. 3, (2013),pp.341-345.

[15] Huang Xiaoqing,Lu lifen,He Tinghui.Mechanics of materials.Guangzhou:South China University of Technology Press,(2011):162-170.

[16] Xiang Chao,Bu Xiongzhu,Qi Keyu,et al.Modeling and compensation of the background eddy-current magnetic field of a high-spin projectile.Journal of Harbin Engineering University, vol. 35, no. 4, (2014),pp.458-462.

[17] Zieve P B. Low voltage electro-impulse de-ic, AIAA-88-0021,(1988).

\section{Authors}

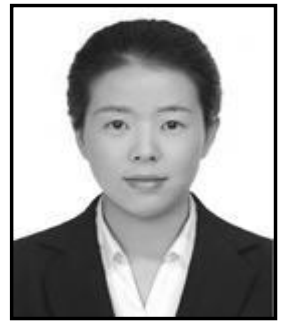

Yuanyuan XU is currently a Ph.D. candidate in the College of Automation at Nanjing University of Aeronautics and Astronautics. Her research interest is mainly in the area of electromechanical system, Avionics system and avionics data bus. She has published several research papers in scholarly journals in the above research. 